Bundesgesundheitsbl 2015 · 58:641-650

DOI 10.1007/s00103-015-2152-3

Online publiziert: 1. April 2015

c) Springer-Verlag Berlin Heidelberg 2015

\section{Prävention und Kontrolle Katheter-assoziierter Harnwegsinfektionen}

\section{Vorbemerkung}

$\$ 23$ Abs. 1 Satz 3 Infektionsschutzgesetz (IfSG) verpflichtet die Kommission für Krankenhaushygiene und Infektionsprävention (KRINKO), ihre Empfehlungen aufgrund neuer infektionsepidemiologischer Erkenntnisse stetig weiterzuentwickeln. Außerdem wurden die Kategorien, mit denen die Evidenz einer Empfehlung beschrieben wird, im Jahr 2010 neu gefasst [1]. Beide Regularien waren ein wichtiger Anlass, die bisherige Empfehlung zur Prävention Katheter-assoziierter Harnwegsinfektionen zu überarbeiten und insgesamt neu zu fassen. Empfehlungen aus der Veröffentlichung aus dem Jahr 1999, die sich in der täglichen Praxis bewährt haben und den Stand von Wissenschaft und Technik wiedergeben, wurden unverändert übernommen [2].

Häufige Anfragen betrafen:

1. Wie oft muss der Auffangbeutel eines Harnableitungssystems gewechselt werden?

2. Was sind geeignete Mittel zur Desinfektion des Auslasses bei Entleerung des Auffangbeutels, bzw. Wechsel des Auffangbeutels?

Beide Fragen betreffen die sachgerechte Anwendung eines Medizinproduktes. Darüber haben Hersteller in der Gebrauchsanweisung erschöpfend Auskunft zu geben ( $\$ 3$ Nr. 10 MPG; $\$ 2$ Abs. 5 MPBetreibV). Deshalb können im Rahmen einer KRIN$\mathrm{KO}$-Empfehlung keine eigenen bzw. von den jeweiligen Herstellerangaben abweichende Empfehlungen erfolgen, da sie materielles Recht nicht ersetzen können [3].

\subsection{Bezug zu anderen KRINKO-Empfehlungen}

Auch andere Empfehlungen der Richtlinie für Krankenhaushygiene und Infektionsprävention enthalten ergänzende Hinweise zur Umsetzung der nachfolgend getroffenen Aussagen; dies sind namentlich: Händehygiene [4], Anforderungen der Krankenhaushygiene und des Arbeitsschutzes an die Hygienebekleidung und persönliche Schutzausrüstung [5] und Mitteilung der Kommission für Krankenhaushygiene und Infektionsprävention zur Surveillance (Erfassung und Bewertung) von nosokomialen Infektionen (Umsetzung von $\$ 23$ IfSG) [6].

\subsection{Kategorien für die Empfehlungen der KRINKO}

Die Definition der Kategorien, die den KRINKO-Empfehlungen zu Grunde gelegt werden, sind nachfolgend in $\bullet$ Tab. 1 aufgeführt.

\subsection{Internationale Leitlinien zur Prävention Katheter-assozi- ierter Harnwegsinfektionen}

Die Autorinnen Conway und Larson haben 2012 acht englischsprachige Empfehlungen zur Prävention Katheter-assoziierter Harnwegsinfektionen (catheter-associated urinary tract infections $=$ CAUTI) verglichen und konstatieren eine bemerkenswerte Konstanz von Empfehlungen über 30 Jahre [7-9]. Weiter stellen sie fest, dass es nach wie vor zu speziellen Fragestellungen nur wenige Studien mit heterogenen Ergebnissen gibt, und in der SHEA (The Society for Healthcare Epidemiology of America)/IDSA (Infectious Diseases Society of America)-Empfehlung aus dem Jahr 2008 sind nur sieben konkrete Handlungsempfehlungen enthalten, die auf mehr als einer randomisierten, kontrollierten Studie basieren [10].

Neuere Publikationen empfehlen $u$. a. eine Bündelung von Einzelmaßnahmen [11]. Weitere Empfehlungen zur Prävention von CAUTI finden sich auch in den aktualisierten Empfehlungen (epic3) von

Tab. 1 Kategorien in der Richtlinie für Krankenhaushygiene und Infektionsprävention (2010)

Kategorie IA: Diese Empfehlung basiert auf gut konzipierten systematischen Reviews oder einzelnen hochwertigen randomisierten kontrollierten Studien

Kategorie IB: Diese Empfehlung basiert auf klinischen oder hochwertigen epidemiologischen Studien und strengen, plausiblen und nachvollziehbaren theoretischen Ableitungen

Kategorie II: Diese Empfehlung basiert auf hinweisenden Studien/Untersuchungen und strengen, plausiblen und nachvollziehbaren theoretischen Ableitungen

Kategorie III: Maßnahmen, über deren Wirksamkeit nur unzureichende oder widersprüchliche Hinweise vorliegen, deshalb ist eine Empfehlung nicht möglich

Kategorie IV: Anforderungen, Maßnahmen und Verfahrensweisen, die durch allgemein geltende Rechtsvorschriften zu beachten sind 
Loveday et al. [12] und in der SHEA/IDSA-Empfehlung von Lo et al. aus dem Jahr 2014 [13].

Die von Conway und Larson gesichteten Empfehlungen stimmen weitgehend (über 30 Jahre) in den folgenden Punkten überein:

- Jede Anlage eines Harnwegskatheters bedarf einer strengen, ärztlichen Indikationsstellung.

- Die Technik beim Legen von Harnwegskathetern und bei der Katheterpflege erfordert ebenso wie die Erkennung von Katheter-assoziierten Komplikationen eine regelmäßige Schulung.

- Das Legen eines Katheters erfolgt unter aseptischen Bedingungen.

- Es sind nur sterile und geschlossene Harnableitungssysteme zu verwenden.

- Katheter sollen so früh wie möglich entfernt werden.

\section{Risikocharakterisierung}

\subsection{Epidemiologische Aspekte}

Harnwegsinfektionen gehören in Krankenhäusern mit einem Anteil von 23,2\% neben den postoperativen Wundinfektionen $(24,3 \%)$, den unteren Atemwegsinfektionen $(21,7 \%)$, gefolgt von Clostridium difficile-Infektionen $(6,4 \%)$ und der primären Sepsis $(5,7 \%)$ zu den häufigsten nosokomialen Infektionen [14, 15]. In $80 \%$ der Fälle sind nosokomiale Harnwegsinfektionen mit einem Katheter assoziiert [16], wobei zwischen $12 \%$ und $16 \%$ aller Krankenhauspatienten im Verlauf ihres Krankenhausaufenthaltes einen Blasenverweilkatheter erhalten $[10,17,18]$. Besonders hoch ist die Prävalenz von Harnwegskathetern auf Intensivstationen [19, 20]. Nach den Daten des Krankenhaus-Infektions-Surveillance-Systems KISS (Infektionssurveillance im Modul ITS-KISS, Berechnungszeitraum Januar 2009 bis Dezember 2013) betrug die Harnwegskatheter-Anwendungsrate (ab Januar 2011) auf diesen Stationen 81,7\% (gepoolter arithmetischer Mittelwert). Die Inzidenzdichte der Harnwegsinfektionen belief sich auf Intensivstationen im Zeitraum 2011 bis 2013 auf 0,93 pro
1000 Harnwegskathetertage [21], auf Normalstationen lag der Wert bei 3,79 Harnwegsinfektionen pro 1000 Harnwegskathetertage [22].

Die tägliche Inzidenz einer neu erworbenen Bakteriurie bei transurethral katheterisierten Patienten liegt zwischen $3 \%$ und $10 \%$, sodass nach 30 Tagen bei der Mehrzahl der Patienten eine Bakteriurie nachzuweisen ist [23]. Der transurethrale Dauerkatheter ist somit der bedeutendste Risikofaktor für eine aszendierende Harnwegsinfektion wie Urethritis, Prostatitis, Epididymitis, Zystitis, Pyelonephritis, Bakteriämie und Urosepsis [24].

Die Vermeidung von Harnröhrenstrikturen und subvesikalen Infektionen sowie die problemlose Kontrolle von Spontanmiktion und Restharn sind entscheidende Vorteile des suprapubischen Katheters.

Etwa $17 \%$ aller Fälle von nosokomialen Bakteriämien sind Folge von Katheter-assoziierten Infektionen der Harnwege. Klinisch relevante Bakteriämien im Sinne einer Urosepsis sind mit einer Mortalität von $10 \%$ verbunden [9]. Insgesamt verlängern die CAUTI die Dauer der stationären Behandlung und erhöhen die Morbidität und Mortalität sowie die Behandlungskosten $[25,26]$.

Bei der klinischen Bewertung von nosokomialen, Katheter-assoziierten Harnwegsinfektionen ist zwischen einer

- asymptomatischen Bakteriurie und einer

- symptomatischen Harnwegsinfektion

zu unterscheiden $[27,28]$. Während die symptomatische Infektion grundsätzlich einer Behandlung bedarf, gilt dies für die asymptomatische Bakteriurie nicht. So entwickeln $90 \%$ der Patienten mit einer asymptomatischen Bakteriurie keine symptomatische Infektion und bei weniger als $5 \%$ kommt es zu einer Bakteriämie [9]. Die antibiotische Behandlung asymptomatischer Bakteriurien bei liegendem Katheter bedarf deshalb einer strengen Indikationsstellung und sollte nur in begründeten Ausnahmefällen (z. B. in der Schwangerschaft und/oder unter Immunsuppression) vorgenommen werden [7].

\subsection{Erregerspektrum}

Für die Jahre 2009 bis 2013 wurden entsprechend dem KISS als Erreger Harnwegskatheter-assoziierter Harnwegsinfektionen E. coli $(43,6 \%$, davon beträgt der ESBL-Anteil 11,8\%), Enterococcus spp. (23,0\%), P. aeruginosa (10,7\%), Klebsiella spp. (10,3\%), Proteus spp. (9,6\%), S. saprophyticus $(2,2 \%)$ und S. aureus $(3,2 \%)$ genannt [29].

\subsection{Infektionsquelle}

Erregerreservoire der CAUTI sind die endogene Flora des Gastrointestinaltraktes, des Urogenitaltraktes und der Perianalregion des Patienten. Weitere Infektionsquellen können bei Missachtung der Regeln einer aseptischen Vorgehensweise, z. B. durch kontaminierte Medizinprodukte, entstehen [8]. Den Harntrakt erreichen die Erreger dabei entweder extraluminal, entlang der sogenannten mucopurulenten Membran zwischen dem Katheter und der Harnröhrenwand oder intraluminal aszendierend bei unsachgemäßer Diskonnektion des geschlossenen Harndrainagesystems [30, 31].

\subsection{Risikofaktoren}

Die Definition von Risikofaktoren für die Entstehung von CAUTI ist eine wesentliche Grundvoraussetzung für die erfolgreiche Einleitung von Präventionsmaßnahmen $[9,11]$.

Als solche Risikofaktoren sind beschrieben [32-39]:

- die Dauer der Katheterisierung

- eine eingeschränkte Immunität

- ein fortgeschrittenes Lebensalter (>50 Jahre)

- das weibliche Geschlecht

- die Diskonnektion des geschlossenen Harndrainagesystems

- die Missachtung von Hygieneregeln bei der Katheterisierung und Katheterpflege

- ein Diabetes mellitus

- eine Niereninsuffizienz

In Abhängigkeit von Faktoren, wie z. B. der Dauer der Katheterisierung, der Harnzusammensetzung, der Diurese und des 
Kathetermaterials bildet sich entlang der Katheteroberfläche insbesondere im Innenlumen ein Biofilm, der zu einer Erregerpersistenz führen kann, die sich durch eine Antibiotikatherapie nicht erfolgreich behandeln lässt [40-42].

\section{Prävention}

\subsection{Sozio-ökonomische Aspekte}

Der Prävention nosokomialer, Katheterassoziierter Harnwegsinfektionen kommt aus individueller Patientensicht als auch aus sozio-ökonomischen Aspekten eine herausragende Bedeutung zu. In den USA schätzt man die zusätzlichen Kosten für eine Episode dieser Infektion auf 600 Dollar und für eine Episode einer Katheter-assoziierten Bakteriämie auf 2.800 Dollar [23]. Entsprechende Daten zu den aufgeführten zusätzlichen Kosten liegen für Deutschland nicht vor.

\subsection{Präventionspotential}

Es ist davon auszugehen, dass bis zu $70 \%$ aller CAUTI durch geeignete Präventionsmaßnahmen verhindert werden könnten $[31,43]$. Voraussetzung für eine erfolgreiche Prävention ist eine stringente Surveillance im Hinblick auf die Prävalenz [23, 44]. $\mathrm{Zu}$ den wirksamsten Präventionsmaßnahmen gehören in diesem Zusammenhang die Vermeidung unnötiger Katheterisierungen, die tägliche Überprüfung der Indikation und in den Fällen wo ein Katheter unvermeidbar ist, die Beschränkung der Liegedauer auf ein $\mathrm{Mi}$ nimum [31, 45-51]. Als besonders wirksam bezüglich der Prävention hat sich die strikte Umsetzung verschiedener Interventionsbündel erwiesen [33, 52-56].

Verschiedene Publikationen zur Wirksamkeit von Interventionsbündeln haben eine Reduktion der Raten von CAUTI bzw. eine Reduktion der Anwendungsraten und/oder Anwendungsdauer von Harnwegskathetern gezeigt $[45,52,55$, 57-64]. In den unterschiedlichen Settings/Risikobereichen wurden jeweils unterschiedliche Maßnahmenbündel eingesetzt. Im informativen Anhang zu dieser Empfehlung werden drei Studien aus verschiedenen Ländern und in verschie- denen Settings beispielhaft vorgestellt [ 55 , $58,59]$.

Das vorrangige Ziel der nachfolgenden Empfehlungen besteht darin, die Häufigkeit infektiöser Komplikationen im Zusammenhang mit dem Katheterismus der Harnblase zu minimieren.

\section{Empfehlung der Kommission}

\subsection{Indikationsstellung für eine Katheterisierung, Liegedauer des Katheters und Interventionsbündel}

Die strenge, medizinisch begründete Indikationsstellung und die Beschränkung der Liegedauer eines Katheters auf das medizinisch notwendige Minimum gehören zu den effektivsten und damit wesentlichen Maßnahmen zur Prävention von CAUTI. Die Implementierung von Kombinationen verschiedener Einzelmaßnahmen durch Interventionsbündel ist inzwischen etabliert [9, 11, 46, 65-71].

Als Beispiele für medizinisch begründete Anwendungen eines Katheters gelten [9]:

- akuter Harnverhalt

- Notwendigkeit der Bilanzierung bei schwer kranken Patienten

- Patienten mit urologischen Operationen

- Förderung der Wundheilung im Bereich des äußeren Genitale bei Harninkontinenz

- mehrstündige Operationen mit hohem Flüssigkeitsumsatz

- Palliative Therapie am Lebensende (auf Wunsch des Patienten).

Als Beispiele für unnötige Anwendungen eines Katheters gelten dagegen [11]:

- die Verordnung nur aufgrund einer Harninkontinenz des Patienten und

- eine Verlängerung der Katheterliegedauer, z. B. bei Patienten nach chirurgischen Eingriffen oder nach Abschluss der intensivmedizinischen Überwachung/Bilanzierung.

\section{Die Kommission empfiehlt:}

- jede Anwendung eines Blasenkatheters von einer strengen, medizinisch begründeten und ärztlich angeord- neten Indikation abhängig zu machen und diese zu dokumentieren (Kat. IB),

- die Liegedauer eines Blasenverweilkatheters stets auf ein erforderliches Minimum zu beschränken (Kat. IB),

- das Weiterbestehen einer Indikation für eine Katheterdrainage täglich ärztlich zu überprüfen und zu dokumentieren (Kat. IB),

- in Einrichtungen ohne ständige ärztliche Anwesenheit entsprechende ärztliche Anordnungen regelmäßig zeitnah einzuholen (Kat. II),

- die Einführung von sog. Interventionsbündeln, wobei sich die Verwendung von Checklisten in einigen Studien bewährt hat (Kat. IB).

Bezüglich spezifischer Aspekte bei Patienten mit neurogenen Blasenfunktionsstörungen wird auf die entsprechende Leitlinie der Deutschen Gesellschaft für Urologie aus dem Jahre 2014 verwiesen [72].

\subsection{Schulung von Personal und Beachtung aseptischer Arbeitsweisen}

Die strenge Beachtung der Regeln der Basishygiene und die regelmäßige Schulung von ärztlichem und pflegerischem Personal ist zur erfolgreichen Prävention von Katheter-assoziierten Infektionen unerlässlich $[9,11,73-80]$.

\section{Die Kommission empfiehlt:}

- Personen, welche die Katheterisierung vornehmen, zum aseptischen Vorgehen, zum Umgang mit dem liegenden Katheter und in der Erkennung Katheter-assoziierter Komplikationen regelmäßig zu schulen (Kat. II),

- vor und nach jeder Manipulation am Blasenverweilkatheter oder Drainagesystem eine hygienische Händedesinfektion vorzunehmen (Basishygiene),

- die Katheterisierung aseptisch vorzunehmen (Basishygiene),

- sterile Handschuhe, steriles Abdeckmaterial (Lochtuch), sterile Tupfer, ggf. eine Pinzette zur aseptischen Katheterinsertion, ein Schleimhautantiseptikum für die Dekontamination 
der Harnröhrenöffnung und ihrer Umgebung und steriles Gleitmittel zu verwenden (Basishygiene),

- um Urothelschäden zu minimieren, die Katheterstärke an die Maße des Meatus urethrae externus anzupassen ( $\leq 18$ Charr. beim Erwachsenen),

- den Ballon eines Blasenverweilkatheters mit sterilem Aqua dest. nach Herstellerangaben, oder besonders bei kleinen Blockvolumina vorzugsweise mit einer sterilen 8-10\%igen Glycerin-Wasserlösung zu füllen und eine Überblockung zu vermeiden (Letztere minimiert das Risiko einer Verklebung des Blockkanals und dichtet die Membranporen des Ballonblocks von innen ab und beugt so einem spontanen Entblocken vor.),

- nur geschlossene Ableitungssysteme einzusetzen, welche die hygienischen Anforderungen an die Probeentnahmestelle für bakteriologische Untersuchungen, an die Rückflusssperre, das Luftausgleichsventil, den Ablassstutzen sowie an das Ablassventil erfüllen (DIN EN ISO 8669-2: 199704) (Kat. II),

- durchhängende Schlaufen des Ableitungssystems, in denen der Urin länger verweilt, zu vermeiden $[81,82]$ (Kat.II),

- den Auffangbeutel vor jedem Transport des Patienten zu leeren, vor allem wenn eine Umlagerung des Patienten (z. B. auf eine Trage oder auf einen OP-Tisch) erforderlich ist [81, 82] (Kat.II),

- beim Entleeren des Drainagebeutels auf Spritzschutz und die Verhinderung des Nachtropfens (an der Rückstecklasche) zu achten (Kat. II),

- den Katheter und den Drainageschlauch grundsätzlich, außer bei spezifischen urologischen Indikationen, nicht zu diskonnektieren (Kat. II). Ausnahmen können für mobile Patienten beim Wechsel von einem Beinbeutel und/oder Nachtbeutel bestehen. Dabei sind folgende Maßnahmen zur Desinfektion und Asepsis einzuhalten:

- die Konnektionsstelle vorher mit einem alkoholischen Präparat zu wisch- oder sprühdesinfizieren, wenn eine Diskonnektion nicht zu vermeiden ist (Kat. II),

- bei Diskonnektion eine erneute Verbindung von Katheter und Konus des Drainageschlauches nur unter aseptischen Kautelen nach Wisch- oder Sprühdesinfektion mit einem alkoholischen Präparat (z. B. einem Hautdesinfektionsmittel) durchzuführen (Kat. II),

- den Auffangbeutel rechtzeitig zu entleeren, bevor der Harn mit der Rückflusssperre in Kontakt kommt (Kat. II) und dabei aus Gründen des Personalschutzes keimarme Einmalhandschuhe zu tragen (Kat. IV),

- bei der Harnentsorgung den Ablassstutzen nicht mit dem Auffanggefäß in Kontakt kommen zu lassen (Kat. II),

- das Auffanggefäß patientenbezogen einzusetzen und nach der Entleerung desinfizierend zu reinigen (Kat. II).

\subsection{Pflege des Meatus urethrae und des Katheters}

Die Kommission empfiehlt:

- die Reinigung des Genitales mit Trinkwasser und Seifenlotion ohne Zusatz antiseptischer Substanzen im Rahmen der normalen, täglichen Körperpflege vorzunehmen (Kat. II),

- Inkrustationen des Katheters im Bereich der Urethraöffnung schonend zu entfernen (Kat. II),

- eine Abknickung der Harnableitung (Katheter und Drainageschlauch) zu vermeiden (Kat. II),

- den Auffangbeutel frei hängend ohne Bodenkontakt und stets unter Blasenniveau anzubringen (Kat. II).

\subsection{Wechselintervalle}

Ein regelmäßiger Wechsel des Katheters oder Auffangbeutels nach festen Intervallen hat bezüglich der Prävention von Katheter-assoziierten Infektionen keine Vorteile gebracht $[83,84]$.

\section{Die Kommission empfiehlt:}

- den Blasenverweilkatheter aus Gründen der Infektionsprävention nicht routinemäßig in festen Intervallen zu wechseln. Bei Infektion, Inkrustation, Obstruktion, Verschmutzung, technischem Defekt des Katheters/Drainagesystems usw. erfolgt der Wechsel nach individuellen Gesichtspunkten und nach ärztlicher Indikationsstellung (Kat. II),

- keinen routinemäßigen Wechsel des Harnwegskatheters:

- als festen Bestandteil einer Sepsisevaluation [58]

- bei Übernahme eines Patienten mit Harnwegskatheter aus einem Pflegeheim [23].

- Im Falle einer Katheter-assoziierten Harnwegsinfektion ist der Katheter wenn möglich zu Beginn (bzw. im Verlauf) der antibiotischen Therapie ganz zu entfernen oder zu wechseln $[8,27,85]$ (Kat II).

- beim Wechseln eines Blasenverweilkatheters stets das gesamte Harnableitungssystem mit auszutauschen (Kat. II).

\subsection{Gewinnung von Harnproben und mikrobiologische Überwachung}

\section{Die Kommission empfiehlt:}

- für die mikrobiologische Diagnostik den Harn nach vorheriger Wischdesinfektion mit einem alkoholischen Präparat nur aus der dafür vorgesehenen patientennahen Entnahmestelle am Drainagesystem zu entnehmen (Kat. II),

- eine bakteriologische Harnuntersuchung dauerkatheterisierter Patienten grundsätzlich nur bei klinischer Symptomatik, vor Operationen am Harntrakt oder aus epidemiologischen Gründen durchzuführen (Kat. II).

\subsection{Antibiotische Prophylaxe und Spülungen der Harnblase}

Eine antibiotische Prophylaxe, die Verwendung von antiseptischen Substanzen im Harnableitungssystem und die regelmäßige Spülung der Harnblase haben bezüglich der Prävention von Harnwegsinfektionen nach dem Legen eines Katheters keine Vorteile gebracht $[9,11,65,86-90]$. 
Die Kommission empfiehlt:

- keine prophylaktische Anwendung von Antibiotika bei Legen eines Dauerkatheters bzw. während der Katheterliegedauer (Kat. II),

- außer bei speziellen urologischen Indikationen (z. B. nach transurethralen Operationen) auf regelmäßige Spülungen der Harnblase bei liegendem Katheter zu verzichten (Kat. II),

- keine Instillation von antiseptischen oder antimikrobiellen Substanzen in das Harndrainagesystem zur Behandlung und Prävention Katheter-assoziierter Infektionen (Kat. II).

\section{Die Kommission stellt fest:}

Es kann keine Empfehlung bezüglich der Ansäuerung des Urins im Sinne der Infektionsprophylaxe abgegeben werden (Kat. III).

\section{7 "Blasentraining" (Abklemmen des Katheters vor der Entfernung)}

Ein sog. Blasentraining vor Entfernung eines Katheters ist grundsätzlich unnötig und erhöht möglicherweise die Häufigkeit von Katheter-assoziierten Infektionen $[9,11,65,91]$.

\section{Die Kommission empfiehlt:}

- auf ein Blasentraining vor Entfernung des Katheters grundsätzlich zu verzichten (Kat. II).

\subsection{Auswahl der Harndrainageform (intermittierender Katheterismus vs. transurethraler oder suprapubischer Blasenverweilkatheter)}

Grundsätzlich ist zwischen

- transurethralen Blasenverweilkathetern,

- suprapubischen Blasenverweilkathetern, die mit einem geschlossenen Auffangsystem verbunden sind und

- der intermittierenden transurethralen (Selbst-) Katheterisierung

zu unterscheiden $[8,24]$.

Zur Prävention von Katheter-assoziierten Infektionen ist ein intermittieren- der Katheterismus, wo immer dies praktikabel ist, dem Blasenverweilkatheter vorzuziehen. $\mathrm{Zu}$ der Frage, ob eine suprapubische Harnableitung gegenüber der transurethralen Ableitung zu einer signifikanten Verringerung von Infektionen beitragen kann, liegen widersprüchliche Ergebnisse vor [7-10, 87, 92, 93]. Zur Schonung der Harnröhre kann es sinnvoll sein, bei absehbarer Langzeitkatheterisierung einen suprapubischen Katheter vorzuziehen [65, 87, 92, 94-97].

\section{Die Kommission empfiehlt:}

- vor jeder Anwendung eines Blasenverweilkatheters zu überprüfen, ob alternativ nicht ein aseptischer, intermittierender Einmal- (Selbst-) Katheterismus in Frage kommt (Kat. II),

- eine Schulung des Patienten bzw. der Pflegenden vorzunehmen und möglichst durch geeignetes Informationsmaterial zu ergänzen (Kat. II),

- eine suprapubische Katheterdrainage zur Umgehung und Schonung der Harnröhre bei längerfristig Katheterisierten und nach größeren operativen Eingriffen, insbesondere im kleinen Becken und am Genitale, in Betracht zu ziehen (Kat. II).

\subsection{Kathetermaterial bzw. Beschichtung des Katheters}

\subsubsection{Latex vs. Silikon und weitere}

Für den Einmalkatheterismus werden Katheter aus PVC verwendet [98]. Latex ist das Kathetermaterial mit dem höchsten allergenen Potential [99]. Vollsilikon besitzt die höchste Biokompatibilität und -stabilität und ist daher für die transurethrale und suprapubische Langzeitdrainage am besten geeignet [100]. Vollsilikon und Hydrogel-beschichtete Katheter haben Vorteile im Hinblick auf Patientenkomfort und Inkrustationsvermeidung $[98,101]$.

Jahn et al. führten eine zuletzt 2012 aktualisierte Cochrane-Analyse der verfügbaren Studien (Erwachsene) zum Einfluss des Kathetermaterials auf zahlreiche Endpunkte durch [102]. Dabei ergaben sich für den Endpunkt Harnwegsinfektion keine signifikanten Unterschiede in Abhängigkeit vom Kathetermaterial (Latex vs. Silikon vs. PVC). Allerdings han- delt es sich zum großen Teil um Studien mit sehr kleiner Patientenzahl. Weitere Hinweise darauf, dass sich Latexkatheter gegenüber Silikonkathetern bezüglich der Rate Katheter-assoziierter Infektionen unterscheiden, liegen bis heute nicht vor $[13,103,104]$.

\section{Die Kommission stellt fest:}

- Aus Gründen der Infektionsprävention kann keine Empfehlung zur bevorzugten Verwendung bestimmter Kathetermaterialien gegeben werden (Kat. III).

\subsubsection{Hydrogel-beschichteter Katheter vs. Silikon}

Hydrogel-beschichtete Katheter scheinen bei langfristiger Anwendung besser toleriert zu werden, es gibt jedoch keinen Hinweis auf einen Vorteil aus Gründen der Infektionsprävention [102, 105].

\section{Die Kommission stellt fest:}

- Aus Gründen der Infektionsprävention kann keine Empfehlung zur bevorzugten Verwendung von Hydrogel-beschichteten Kathetern anstelle von nicht beschichteten Kathetern gegeben werden (Kat. III).

\subsubsection{Antimikrobiell beschichtete Harnwegskatheter}

Johnson et al. fassten 2006 zwölf Studien zu Silber-beschichteten Latexkathetern $(n=9)$ oder mit Nitrofurazon-beschichteten Kathetern $(n=3)$ in einer systematischen Analyse zusammen und stellten fest, dass alle Studien erhebliche methodische Mängel aufwiesen. Die wichtigste Limitation war, dass in diesen Studien nicht die symptomatische Harnwegsinfektion sondern ausschließlich eine Katheter-assoziierte Bakteriurie als Endpunkt untersucht wurde [106]. Karchmer et al. [107] untersuchten in einer prospektiven cross-over Studie ${ }^{1}$ den Nutzen Silber-imprägnierter und Hydrogel-beschichteter Harnwegskatheter auf die Infektionsrate und fanden einen signifikanten Vorteil für den beschichteten Katheter bei aus-

\footnotetext{
1 Randomisierte Zuordnung des jeweiligen Kathetertyps zu den Studienstationen für jeweils 6 Monate; Wechsel der Zuordnung nach einer "wash-out“ Phase von einem Monat.
} 
schließlicher Betrachtung der „Normalstationen"ohne ICU (2,13 vs. 3,12 Infektionen pro 100 Katheter; RR 0,68 CI95 0,54$0,86 ; p<0,01)$. In beiden Gruppen traten die Infektionen nach einer medianen Liegedauer von 9 Tagen auf. In einer methodisch gut konzipierten, prospektiven multizentrischen Studie ${ }^{2}[103,104]$ zum infektionspräventiven Nutzen antimikrobiell beschichteter Harnwegskatheter verglichen die Autoren Silikonkatheter, die mit Silber oder einem Antibiotikum (Nitrofurazon) beschichtet waren, mit einer Kontrollgruppe (Teflon-beschichtete Latexkatheter). In Bezug auf die Rate von symptomatischen Harnwegsinfektionen, die eine antibiotische Therapie erforderlich machten, ergaben sich in den 6 Wochen Beobachtungszeit nach Anlage des Katheters keine signifikanten Unterschiede.

\section{Die Kommission stellt fest:}

- Auf Grundlage der verfügbaren Studiendatenlage kann keine Empfehlung zur Verwendung antimikrobiell beschichteter Katheter gegeben werden (Kat. III).

\section{Informativer Anhang}

Im Folgenden werden drei Studien aus verschiedenen Ländern und in verschiedenen Settings beispielhaft vorgestellt. Bei diesen waren die Interventionsbündel eingebettet u. a. in Trainings, Qualitätskontroll- und Surveillance-Programme:

Marra et al. [59] untersuchten in einer ICU (Intensivpflegestation) und zwei SDUs (step-down units, intermediäre Stationen in einem Krankenhaus in Brasilien den Effekt von Interventionen, die zur Reduktion der Inzidenz von Katheter-assoziierten Harnwegsinfektionen implementiert wurden. Dabei wurde u. a. zusätzlich ein Interventionsbündel, bestehend aus den folgenden Komponenten, eingesetzt:

- Zusammenstellung eines „Harnwegskatheter-Insertions-Wagens “

- Händehygiene

- Antiseptik der Haut und des Meatus urethrae mit Chlorhexidin

- Sterilfeld und sterile Handschuhe

2 Jugendliche $\geq 16$ Jahre und Erwachsene, Liegedauer des Katheters $\leq 14$ Tage, 24 Krankenhäuser in Großbritannien.
- Nur ein Insertionsversuch pro Katheter zulässig (d. h. Einsatz eines neuen Katheters für jeden Versuch)

- Adäquate Befüllung des Blasenverweilkatheter-Ballons

- Tägliche Überprüfung der Notwendigkeit für einen Harnwegskatheter und sofortige Entfernung, wenn er nicht länger benötigt wird.

Die Rate Katheter-assoziierter Harnwegsinfektionen in der ICU konnte signifikant von 7,6 vor Intervention auf 5,0 pro 1000 Kathetertage nach Intervention reduziert werden und in den SDUs von 15,3 vor Intervention auf 12,9 pro 1000 Kathetertage nach Intervention.

In einer Studie von Rosenthal et al. [55] in 10 pädiatrischen ICUs (PICUs) in sechs Entwicklungsländern kam es nach der Implementierung eines multidimensionalen Infektions-Kontroll-Ansatzes zu einer Reduktion der Raten Katheter-assoziierter Harnwegsinfektionen um $57 \%$ (von 5,9 auf 2,6 pro 1000 Kathetertage). Dieser Ansatz beinhaltete u. a. ein „Praxisbündel“ zur Prävention Katheter-assoziierter Harnwegsinfektionen und bestand aus folgenden Interventionen:

- Schulung und Training zu Insertion und Pflege von Blasenverweilkathetern, Alternativen zu Blasenverweilkathetern und Vorgehensweisen bei Katheterinsertion, -management und -entfernung

- Insertion von Harnwegskathetern nur wenn notwendig und Entfernung der Katheter, wenn diese nicht notwendig sind

- Anwendung von Blasenverweilkathetern für perioperative und ausgewählte chirurgische Eingriffe; Monitoring der Urinausscheidung bei kritisch kranken Patienten; Management des akuten Harnverhaltes und der Harnabflussstörung; Unterstützung der Heilung von Dekubitalulzera bei Inkontinenz

- Berücksichtigung anderer Methoden zum Management, einschließlich Urinalkondome oder intermittierende Einmalkatheterisierung, wenn geeignet

- Händehygiene vor Insertion und Manipulation am Harnwegskatheter
- Verwendung des Katheters mit kleinstmöglichem Durchmesser

- Verwendung von Handschuhen, Abdecktuch und Schwämmen; sterile oder antiseptische Lösung zur Reinigung des Meatus urethrae; Packung für den Einzelgebrauch mit sterilem Gleitmittel zur Insertion

- Insertion der Harnwegskatheter unter Anwendung aseptischer Technik und Verwendung steriler Materialien

- Angemessenes Management der Harnwegskatheter, einschließlich sachgemäßer Befestigung des Blasenverweilkatheters, um Verrutschen $\mathrm{zu}$ vermeiden; Erhaltung eines sterilen, kontinuierlich geschlossenen Harnableitungssystems; keine Diskonnektion des Katheters und Drainageschlauchs; Ersetzen des Ableitungssystems unter Anwendung aseptischer Technik und nach Desinfektion der Katheterschlauchverbindung, bei Lücken in der aseptischen Technik, Diskonnektion oder Leckage

- Erhaltung eines ungehinderten Urinabflusses

- Den Auffangbeutel jederzeit unterhalb des Blasenniveaus halten

- Regelmäßige Entleerung des Auffangbeutels und Vermeidung des Kontaktes des Ablassstutzens mit dem Sammelbehälter

- Reinigung des Meatus urethrae als Teil der Routinehygiene

- Surveillance von Katheter-assoziierten Harnwegsinfektionen, Einsatz standardisierter Kriterien zur Identifizierung von Patienten mit Katheter-assoziierten Harnwegsinfektionen und Erfassung von Kathetertagen als Denominator

Titsworth et al. [58] führten eine prospektive Interventionsstudie in einer neurologischen ICU in den USA durch. Nach der Implementierung eines evidenzbasierten Präventionsbündels, gemeinsam mit einem kontinuierlichen Qualitätsverbesserungsprogramm, kam es zu einer signifikanten Reduktion der Anwendungsrate von Harnwegskathetern (von 100\% auf 73,3 \% während der Interventionsphase) sowie der Rate Katheter-assoziierter Harnwegsinfektionen von 13,3 auf 4,0 pro 1000 Kathetertage. 
Tab. 2 Tabellarische Darstellung der beispielhaft vorgestellten Studien

\begin{tabular}{|c|c|c|c|}
\hline & Marra et al. [59] & Rosenthal et al. [55] & Titsworth et al. [58] \\
\hline Länder & Brasilien & $\begin{array}{l}\text { Kolumbien, El Salvador, Indien, Mexiko, } \\
\text { Philippinen, Türkei }\end{array}$ & USA \\
\hline Studiendesign & $\begin{array}{l}\text { Quasi-experimentelle Studie mit } \\
\text { unterbrochener Zeitreihe }\end{array}$ & $\begin{array}{l}\text { Vorher-nachher prospektive Kohorten- } \\
\text { studie }\end{array}$ & $\begin{array}{l}\text { Prospektive Interven- } \\
\text { tionsstudie }\end{array}$ \\
\hline Setting & $\begin{array}{l}\text { Eine medizinisch-chirurgische ICU } \\
\text { und } 2 \text { SDUs in einem Krankenhaus }\end{array}$ & $\begin{array}{l}10 \text { PICUs in } 10 \text { Krankenhäusern (Mit- } \\
\text { glieder des International Nosocomial } \\
\text { Infection Control Consortium (INICC)) }\end{array}$ & $\begin{array}{l}\text { Neurologische ICU in } \\
\text { einem Krankenhaus }\end{array}$ \\
\hline \multicolumn{4}{|l|}{ Bündelfaktoren } \\
\hline Schulung & $(\mathrm{X})$ & $x$ & $\mathrm{x}$ \\
\hline Strenge Indikationsstellung & $(\mathrm{X})$ & $\mathrm{x}$ & $\mathrm{x}$ \\
\hline Händehygiene & $x$ & $\mathrm{X}$ & $x$ \\
\hline Asepsis & $x$ & $x$ & $x$ \\
\hline Geschlossene Harnableitungssysteme & Keine Information & $\mathrm{x}$ & $\mathrm{x}$ \\
\hline $\begin{array}{l}\text { Erinnerungssysteme („reminder systems")/ } \\
\text { Stopp-Anordnungen („,stop orders") }\end{array}$ & $\mathrm{x}$ & Keine Information & $\mathrm{x}$ \\
\hline Surveillance & $(\mathrm{X})$ & $\mathrm{x}$ & $(\mathrm{X})$ \\
\hline Prüfung der Compliance & $\begin{array}{l}\text { Durch Harnwegs-katheter-Check- } \\
\text { liste }\end{array}$ & $\begin{array}{l}\text { Monitoring der Durchführung der } \\
\text { Händehygiene und anderer Infektions- } \\
\text { kontrollmaßnahmen }\end{array}$ & $\begin{array}{l}\text { Durch tägliche Katheter- } \\
\text { visiten („,Foley Rounds") }\end{array}$ \\
\hline \multicolumn{4}{|l|}{ Ergebnis } \\
\hline $\begin{array}{l}\text { Signifikante Reduktion der Rate Katheter- } \\
\text { assoziierter Harnwegsinfektionen }\end{array}$ & $\mathrm{Ja}$ & Ja & $\mathrm{Ja}$ \\
\hline
\end{tabular}

Das Präventionsbündel bestand aus:

\section{Vermeidung einer Katheterinsertion} (strenge Indikationsstellung)

- Entwicklung von Kriterien für die Indikation einer Katheterinsertion

- Grundsätzliche Entfernung des Harnwegskatheters postoperativ, wenn nicht explizit anders angeordnet

- Prüfung alternativer Methoden (intermittierende Einmalkatheterisierung in Verbindung mit Blasensonographie als bevorzugte Methode gegenüber Blasenverweilkatheter)

\section{Produkt-Standardisierung}

- Antimikrobielle Katheter

- Geschlossene Systeme

- Einsatz eines Stabilisierungssystems zur Katheterbefestigung am Bein

\section{Aufrechterhaltung der Kathetersterili-} tät (Asepsis)

- Schulung des Pflegepersonals

- Asepsis bei Katheteranlage und Erhaltung der Kathetersterilität während der Anwendungsdauer
- Auffangbeutel unterhalb des Blasenniveaus, auch während des Transports, und routinemäßige Entleerung

- Befestigung der Auffangbeutel an der der Tür zugewandten Bettseite

- Vermeidung von Schlaufenbildung im Schlauchsammelsystem

- Gebrauch individueller Entleerungsbehälter

- Händehygiene

Rechtzeitiger Entfernung der Harnwegskatheter

- Begründung des Katheters durch Auswahl einer der anerkannten Indikationen für eine Katheteranlage auf einem vorgedruckten Verlaufsbogen

- Ziel der Katheterentfernung vier Tage nach Anlage, im weiteren Verlauf der Interventionsstudie nach zwei Tagen, dann nach einem Tag, außer bei fortbestehender Indikation

- Von Pflegekräften gesteuertes Protokoll zur Entfernung

- Tägliche Kathetervisiten („Foley Rounds") zur Überprüfung des Fortbestehens der Indikation und Compliance bezüglich des Präventions- bündels; Katheterentfernung bei fehlender Indikation

Die Darstellung der Interventionsbündel kann nur als Beispiel dienen, da bei der Zusammensetzung der einzelnen Maßnahmen und der Umsetzung eines Bündels die jeweiligen Bedingungen und Gegebenheiten vor Ort berücksichtigt werden sollten.

Aus Gründen der Übersichtlichkeit werden die Einzelmaßnahmen der oben ausführlich beschriebenen Interventionsbündel in der nachfolgenden - Tab. 2 einheitlich sieben übergeordneten Bündelfaktoren zugeordnet.

Die Empfehlungen wurden ehrenamtlich und ohne Einflussnahme kommerzieller Interessengruppen im Auftrag der Kommission für Krankenhaushygiene und Infektionsprävention erarbeitet von Herrn Prof. Dr. Joachim Martius (Leiter der Arbeitsgruppe), Agatharied; Herrn Prof. Dr. Martin Exner, Bonn; Herrn Prof. Dr. Hansjürgen Piechota, Minden; Herrn Prof. Dr. Arne Simon, Homburg/Saar; Frau Anna Triphaus, Bremen; Frau Dr. Martina Kraus-Haas (RKI), Berlin. 


\section{Literatur}

1. Kommission für Krankenhaushygiene und Infektionsprävention (KRINKO) (2010) Die Kategorien in der Richtlinie für Krankenhaushygiene und Infektionsprävention - Aktualisierung der Definitionen. Bundesgesundheitsbl Gesundheitsforsch Gesundheitsschutz 53(7):754-756

2. Kommission für Krankenhaushygiene und Infektionsprävention (KRINKO) (1999) Empfehlungen zur Prävention und Kontrolle Katheter-assoziierter Harnwegsinfektionen. Bundesgesundheitsbl Gesundheitsforsch Gesundheitsschutz 42:806809

3. Kommission für Krankenhaushygiene und Infektionsprävention (KRINKO) (2011) Anforderungen an die Hygiene bei Punktionen und Injektionen. Bundesgesundheitsbl Gesundheitsforsch Gesundheitsschutz 54(9):1135-1144

4. Kommission für Krankenhaushygiene und Infektionsprävention (KRINKO) (2000) Händehygiene. Bundesgesundheitsbl Gesundheitsforsch Gesundheitsschutz 43:230-233

5. Robert Koch-Institut (2007) Anforderungen der Krankenhaushygiene und des Arbeitsschutzes an die Hygienebekleidung und persönliche Schutzausrüstung. Epid Bull 1:3-4

6. Kommission für Krankenhaushygiene und Infektionsprävention (KRINKO) (2001) Mitteilung der Kommission für Krankenhaushygiene und Infektionsprävention zur Surveillance (Erfassung und Bewertung) von nosokomialen Infektionen (Umsetzung von § 23 IfSG). Bundesgesundheitsbl Gesundheitsforsch Gesundheitsschutz 44(5):523536

7. Conway LJ, Larson EL (2012) Guidelines to prevent catheter-associated urinary tract infection: 1980-2010. Heart Lung 41(3):271-283

8. Hooton TM et al (2010) Diagnosis, prevention, and treatment of catheter-associated urinary tract infection in adults: 2009 international clinical practice guidelines from the infectious diseases society of America. Clin Infect Dis 50(5):625663

9. Gould CV et al (2010) Guideline for prevention of catheter-associated urinary tract infections 2009. Infect Control Hosp Epidemiol 31(4):319-326

10. Lo E et al (2008) Strategies to prevent catheterassociated urinary tract infections in acute care hospitals. Infect Control Hosp Epidemiol 29 (Suppl 1):41-50

11. Chenoweth CE, Gould CV, Saint S (2014) Diagnosis, management, and prevention of catheter-associated urinary tract infections. Infect Dis Clin North Am 28(1):105-119

12. Loveday HP et al (2014) epic3: national evidencebased guidelines for preventing healthcare-associated infections in NHS hospitals in England. J Hosp Infect 86 (Suppl 1):1-70

13. Lo E et al (2014) Strategies to prevent catheterassociated urinary tract infections in acute care hospitals: 2014 update. Infect Control Hosp Epidemiol 35(5):464-479

14. Behnke M et al (2013) Nosocomial infection and antibiotic use - a second national prevalence study in Germany. Deutsches Ärzteblatt International 110(38):627-633

15. Gastmeier P, Geffers C (2008) Nosokomiale Infektionen in Deutschland: Wie viele gibt es wirklich? Eine Schätzung für das Jahr 2006. Dtsch Med Wochenschr 133(21):1111-1115

16. Saint S, Chenoweth CE (2003) Biofilms and catheter-associated urinary tract infections. Infect Dis Clin North Am 17(2):411-432
17. Lewalter K et al (2013) Die katheterassoziierte Harnwegsinfektion - was gibt es Neues? Krankenhaushygiene up2date 8:25-36

18. Weinstein JW et al (1999) A decade of prevalence surveys in a tertiary-care center: trends in nosocomial infection rates, device utilization, and patient acuity. Infect Control Hosp Epidemiol 20(8):543-548

19. Wagenlehner FM et al (2006) Incidence of nosocomial urinary tract infections on a surgical intensive care unit and implications for management. Int J Antimicrob Agents 28 (Suppl 1):86-90

20. Bagshaw SM, Laupland KB (2006) Epidemiology of intensive care unit-acquired urinary tract infections. Curr Opin Infect Dis 19(1):67-71

21. Nationales Referenzzentrum für Surveillance von nosokomialen Infektionen (2014) Infektionssurveillance im Modul ITS-KISS, Referenzdaten Berechnungszeitraum: Januar 2009 bis Dezember 2013; KISS Krankenhaus-Infektions-SurveillanceSystem. http://www.nrz-hygiene.de/fileadmin/ nrz/module/its/200901_201312_ALLE_ITSRef. pdf

22. Nationales Referenzzentrum für Surveillance von nosokomialen Infektionen (2014) Infektionssurveillance im Modul STATIONS-KISS; Referenzdaten Berechnungszeitraum: Januar 2009 bis Dezember 2013. http://www.nrz-hygiene.de/surveillance/kiss/stations-kiss/infektionen/

23. Chenoweth C, Saint S (2013) Preventing catheter-associated urinary tract infections in the intensive care unit. Crit Care Clin 29(1):19-32

24. Tenke P et al (2008) European and Asian guidelines on management and prevention of catheterassociated urinary tract infections. Int J Antimicrob Agents 31 (Suppl 1):68-78

25. Holroyd-Leduc JM et al (2007) The relationship of indwelling urinary catheters to death, length of hospital stay, functional decline, and nursing home admission in hospitalized older medical patients. J Am Geriatr Soc 55(2):227-233

26. Chant $C$ et al (2011) Relationship of catheter-associated urinary tract infection to mortality and length of stay in critically ill patients: a systematic review and meta-analysis of observational studies. Crit Care Med 39(5):1167-1173

27. Trautner BW (2010) Management of catheter-associated urinary tract infection. Curr Opin Infect Dis 23(1):76-82

28. Nationales Referenzzentrum (NRZ) für Surveillance von nosokomialen Infektionen am Institut für Hygiene und Umweltmedizin (2011) Definition nosokomialer Infektionen (CDC-Definitionen) Robert Koch-Institut, Berlin

29. Nationales Referenzzentrum für Surveillance von nosokomialen Infektionen (2014) Infektionssurveillance im Modul STATIONS-KISS Referenzdaten, Berechnungszeitraum: Januar 2009 bis Dezember 2013. http://www.nrz-hygiene.de/surveillance/kiss/stations-kiss/infektionen

30. Shuman EK, Chenoweth CE (2010) Recognition and prevention of healthcare-associated urinary tract infections in the intensive care unit. Crit Care Med 38 (Suppl 8):373-379

31. Rebmann T, Greene LR (2010) Preventing catheter-associated urinary tract infections: an executive summary of the association for professionals in infection control and epidemiology, inc, elimination guide. Am J Infect Control 38(8):644-646

32. Nicolle LE (2012) Urinary catheter-associated infections. Infect Dis Clin North Am 26:13-27
33. Meddings J et al (2014) Reducing unnecessary urinary catheter use and other strategies to prevent catheter-associated urinary tract infection: an integrative review. BMJ Qual Saf 23:277-289

34. van der Kooi TI et al (2007) Incidence and risk factors of device-associated infections and associated mortality at the intensive care in the Dutch surveillance system. Intensive Care Med 33:271278

35. Leone M et al (2003) Risk factors of nosocomial catheter-associated urinary tract infection in a polyvalent intensive care unit. Intensive Care Med 29:1077-1080

36. Tissot $E$ et al (2001) Risk factors for catheter-associated bacteriuria in a medical intensive care unit. Eur J Clin Microbiol Infect Dis 20:260-262

37. Hazelett SE et al (2006) The association between indwelling urinary catheter use in the elderly and urinary tract infection in acute care. BMC Geriatrics 6:15

38. Saint $S$ et al (2006) Risk factors for nosocomial urinary tract-related bacteremia: a case-control study. Am J Infect Control 34:401-407

39. Burke JP, Larsen RA, Stevens LE (1986) Nosocomial bacteriuria: estimating the potential for prevention by closed sterile urinary drainage. Infect Control 7:96-99

40. Jacobsen SM, Shirtliff ME (2011) Proteus mirabilis biofilms and catheter-associated urinary tract infections. Virulence 2(5):460-465

41. Hook AL et al (2012) Combinatorial discovery of polymers resistant to bacterial attachment. Nat Biotechnol 30(9):868-875

42. Niveditha $S$ et al (2012) The isolation and the biofilm formation of uropathogens in the patients with catheter associated urinary tract infections (UTIs). J Clin Diagn Res 6(9):1478-1482

43. Umscheid CA et al (2011) Estimating the proportion of healthcare-associated infections that are reasonably preventable and the related mortality and costs. Infect Control Hosp Epidemiol 32(2):101-114

44. Gastmeier P et al (2011) Benchmarking of urinary tract infection rates: experiences from the intensive care unit component of the German national nosocomial infections surveillance system. J Hosp Infect 78(1):41-44

45. Clarke K et al (2013) Reduction in catheter-associated urinary tract infections by bundling interventions. Int J Qual Health Care 25(1):43-49

46. Fakih MG et al (2012) Reducing inappropriate urinary catheter use: a statewide effort. Arch Intern Med 172(3):255-260

47. Fink $R$ et al (2012) Indwelling urinary catheter management and catheter-associated urinary tract infection prevention practices in Nurses Improving Care for Healthsystem Elders hospitals. Am J Infect Control 40(8):715-720

48. Bernard MS, Hunter KF, Moore KN (2012) A review of strategies to decrease the duration of indwelling urethral catheters and potentially reduce the incidence of catheter-associated urinary tract infections. Urol Nurs 32(1):29-37

49. Huang WC et al (2004) Catheter-associated urinary tract infections in intensive care units can be reduced by prompting physicians to remove unnecessary catheters. Infect Control Hosp Epidemiol 25(11):974-978

50. Jaggi N, Sissodia P (2012) Multimodal supervision programme to reduce catheter associated urinary tract infections and its analysis to enable focus on labour and cost effective infection control measures in a tertiary care hospital in India. J Clin Diagn Res 6(8):1372-1376 
51. Kommission für Krankenhaushygiene und Infektionsprävention (KRINKO) (2005) Infektionsprävention in Heimen. Bundesgesundheitsbl Gesundheitsforsch Gesundheitsschutz 48(9):10611080

52. Andreessen L, Wilde MH, Herendeen P (2012) Preventing catheter-associated urinary tract infections in acute care: the bundle approach. $J$ Nurs Care Qual 27(3):209-217

53. Jansen IA et al (2012) Appropriate use of indwelling urethra catheters in hospitalized patients: results of a multicentre prevalence study. BMC Urol $12: 25$

54. Krein SL et al (2013) Barriers to reducing urinary catheter use: a qualitative assessment of a statewide initiative. JAMA Intern Med 173(10):881886

55. Rosenthal VD et al (2012) Findings of the international nosocomial infection control consortium (INICC), part l: effectiveness of a multidimensional infection control approach on catheter-associated urinary tract infection rates in pediatric intensive care units of 6 developing countries. Infect Control Hosp Epidemiol 33(7):696-703

56. Tambyah PA, Oon J (2012) Catheter-associated urinary tract infection. Curr Opin Infect Dis 25(4):365-370

57. Knoll BM et al (2011) Reduction of inappropriate urinary catheter use at a Veterans Affairs hospital through a multifaceted quality improvement project. Clin Infect Dis 52(11):1283-1290

58. Titsworth WL et al (2012) Reduction of catheterassociated urinary tract infections among patients in a neurological intensive care unit: a single institution's success. J Neurosurg 116(4):911-920

59. Marra AR et al (2011) Preventing catheter-associated urinary tract infection in the zero-tolerance era. Am J Infect Control 39(10):817-822

60. Rosenthal VD et al (2012) Impact of a multidimensional infection control strategy on catheter-associated urinary tract infection rates in the adult intensive care units of 15 developing countries: findings of the International Nosocomial Infection Control Consortium (INICC). Infection 40(5):517-526

61. Leblebicioglu $\mathrm{H}$ et al (2013) Impact of a multidimensional infection control approach on catheter-associated urinary tract infection rates in adult intensive care units in 10 cities of Turkey: international nosocomial infection control consortium findings (INICC). Am J Infect Control 41:885-891

62. Kanj SS et al (2013) Impact of a multidimensional infection control approach on catheter-associated urinary tract infection rates in an adult intensive care unit in Lebanon: International Nosocomial Infection Control Consortium (INICC) findings. Int J Infect Dis 17(9):e686-e690

63. Navoa-Ng JA et al (2013) Impact of an International Nosocomial Infection Control Consortium multidimensional approach on catheter-associated urinary tract infections in adult intensive care units in the Philippines: international nosocomial infection control consortium (INICC) findings. J Infect Public Health 6(5):389-399

64. Venkatram S, Rachmale S, Kanna B (2010) Study of device use adjusted rates in health care-associated infections after implementation of ,bundles" in a closed-model medical intensive care unit. J Crit Care 25(1):174.e11-174.e18

65. Phipps $S$ et al (2006) Short term urinary catheter policies following urogenital surgery in adults. Cochrane Database Syst Rev 2:CD004374
66. Nordqvist $P$ et al (1984) Catheter-free geriatric care. Routines and consequences for clinical infection, care and economy. J Hosp Infect 5:298304

67. Stephan F et al (2006) Reduction of urinary tract infection and antibiotic use after surgery: a controlled, prospective, before-after intervention study. Clin Infect Dis 42:1544-1551

68. Larsen LD et al (1997) Retrospective analysis of urologic complications in male patients with spinal cord injury managed with and without indwelling urinary catheters. Urology 50:418-422

69. Esclarin De Ruz A, Garcia Leoni E, Herruzo Cabrera R (2000) Epidemiology and risk factors for urinary tract infection in patients with spinal cord injury. J Urol 164:1285-1289

70. Saint $S$ et al (2005) A reminder reduces urinary catheterization in hospitalized patients. Jt Comm J Qual Patient Saf 31:455-462

71. Saint S et al (2009) Translating health care-associated urinary tract infection prevention research into practice via the bladder bundle. Jt Comm J Qual Patient Saf 35(9):449-455

72. Böthig R, Geng V, Kurze I (2014) Leitlinie der Deutschen Gesellschaft für Urologie (DGU): Management und Durchführung des Intermittierenden Katheterismus (IK) bei Neurogenen Blasenfunktionsstörungen. AWMF 043/048:1-22

73. Jain M et al (2006) Decline in ICU adverse events, nosocomial infections and cost through a quality improvement initiative focusing on teamwork and culture change. Qual Saf Health Care 15:235-239

74. Reilly L et al (2006) Reducing foley catheter device days in a intensive care unit: using the evidence to change practice. AACN Adv Crit Care 17:272-283

75. Verdier R et al (2006) Impact of an infection control program in an intensive care unit in France. Infect Control Hosp Epidemiol 27:60-66

76. Topal J et al (2005) Prevention of nosocomial catheter-associated urinary tract infections through computerized feedback to physicians and a nurse-directed protocol. Am J Med Qual 20:121-126

77. Rosenthal VD, Guzman S, Safdar N (2004) Effect of education and performance feedback on rates of catheter-associated urinary tract infection in intensive care units in Argentina. Infect Control Hosp Epidemiol 25:47-50

78. Webster J et al (2001) Water or antiseptic for periurethral cleaning before urinary catheterization: a randomized controlled trial. Am J Infect Control 29:389-394

79. Koskeroglu N, et al (2004) The role of meatal disinfection in preventing catheter-related bacteriuria in an intensive care unit: a pilot study in Turkey. J Hosp Infect 56:236-238

80. Matsumoto T et al (1997) Prevention of catheterassociated urinary tract infection by meatal disinfection. Dermatology 195 (Suppl 2):73-77

81. Davis KF et al (2014) Reducing catheter-associated urinary tract infections: a quality-improvement initiative. Pediatrics 134(3):e857-e864

82. Oman KS et al (2012) Nurse-directed interventions to reduce catheter-associated urinary tract infections. Am J Infect Control 40(6):548-553

83. White MC, Ragland KE (1995) Urinary catheterrelated infections among home care patients. J Wound Ostomy Continence Nurs 22:286-290
84. Keerasuntonpong A et al (2003) Incidence of urinary tract infections in patients with short-term indwelling urethral catheters: a comparison between a 3-day urinary drainage bag change and no change regimens. Am J Infect Control 31:9-12

85. Al Mohajer M, Darouiche RO (2013) Prevention and treatment of urinary catheter-associated infections. Curr Infect Dis Rep 15(2):116-23

86. Rogers RG et al (2004) A randomized, doubleblind, placebo-controlled comparison of the effect of nitrofurantoin monohydrate macrocrystals on the development of urinary tract infections after surgery for pelvic organ prolapse and/ or stress urinary incontinence with suprapubic catheterization. Am J Obstet Gynecol 191:182187

87. Niel-Weise B.S et al (2012) Urinary catheter policies for long-term bladder drainage. Cochrane Database Syst Rev 8:CD004201

88. Esposito S et al (2006) A pilot study on prevention of catheter-related urinary tract infections with fluoroquinolones. J Chemother 18:494-501

89. Schiotz HA, Guttu K (2002) Value of urinary prophylaxis with methenamine in gynecologic surgery. Acta Obstet Gynecol Scand 81:743-746

90. van den Broek PJ, Daha TJ, Mouton RP (1985) Bladder irrigation with povidone-iodine in prevention of urinary-tract infections associated with intermittent urethral catheterisation. Lancet 1:563-565

91. Griffiths R Fernandez R (2007) Strategies for the removal of short-term indwelling urethral catheters in adults. Cochrane Database Syst Rev 2:CD004011

92. McPhail MJ, Abu-Hilal M, Johnson C.D (2006) A meta-analysis comparing suprapubic and transurethral catheterization for bladder drainage after abdominal surgery. British Journal of Surgery 93(9):1038-1044

93. Healy EF et al (2012) Suprapubic compared with transurethral bladder catheterization for gynecologic surgery: a systematic review and meta-analysis. Obstet Gynecol 120(3):678-687

94. Johansson I et al (2002) Intermittent versus indwelling catheters for older patients with hip fractures. J Clin Nurs 11:651-656

95. Halleberg Nyman M et al (2013) Intermittent versus indwelling urinary catheterisation in hip surgery patients: a randomised controlled trial with cost-effectiveness analysis. Int J Nurs Stud 50:1589-1598

96. Jannelli ML et al (2007) A randomized controlled trial of clean intermittent self-catheterization versus suprapubic catheterization after urogynecologic surgery. Am J Obstet Gynecol 197(1):72

97. Baan AH et al (2003) The effect of suprapubic catheterization versus transurethral catheterization after abdominal surgery on urinary tract infection: a randomized controlled trial. Dig Surg 20:290-295

98. Piechota H, Pannek J (2007) Katheterdrainage der Harnblase - Stand der Technik und Bedeutung für die Infektionsprävention. Hyg Med 32:336-344

99. Merguerian PA et al (1991) Intraoperative anaphylactic reaction due to latex hypersensitivity. Urology 38(4):301-304

100. Hesse A et al (1994) Experimentelle Untersuchungen zur Inkrustationsneigung und Drainagekapazität von Silikon- und silikonisierten Latexkathetern. Urologe 34:370-374

101. Bach D (1998) Katheter-Inkrustation, Ursachen und Konsequenzen für die Katheterhygiene. Hyg Med 23:404-408 
102. Jahn P, Beutner K, Langer $G$ (2012) Types of indwelling urinary catheters for long-term bladder drainage in adults. Cochrane Database Syst Rev 10:CD004997

103. Pickard R et al (2012) Antimicrobial catheters for reduction of symptomatic urinary tract infection in adults requiring short-term catheterisation in hospital: a multicentre randomised controlled trial. Lancet 380(9857):1927-1935

104. Pickard R et al (2012) Types of urethral catheter for reducing symptomatic urinary tract infections in hospitalised adults requiring short-term catheterisation: multicentre randomised controlled trial and economic evaluation of antimicrobial- and antiseptic-impregnated urethral catheters (the CATHETER trial). Health Technol Assess 16(47):119-197

105. Bull E et al (1991) Single-blind, randomised, parallel group study of the Bard Biocath catheter and a silicone elastomer coated catheter. $\mathrm{Br} J$ Urol 68(4):394-399

106. Johnson JR, Kuskowski M.A, Wilt T. J (2006) Systematic review: antimicrobial urinary catheters to prevent catheter-associated urinary tract infection in hospitalized patients. Ann Intern Med 144(2):116-126

107. Karchmer TB et al (2000) A randomized crossover study of silver-coated urinary catheters in hospitalized patients. Arch Intern Med 160(21):3294-3298 